\title{
Early-age autogenous and drying shrinkage behavior of cellulose fiber-reinforced cementitious materials
}

\author{
Shiho Kawashima*, Surendra P. Shah \\ Northwestern University, Civil and Environmental Engineering, 2145 Sheridan Road, Evanston, IL 60208, USA
}

\section{A R T I C L E I N F O}

\section{Article history:}

Received 12 January 2010

Received in revised form 18 October 2010

Accepted 22 October 2010

Available online 28 October 2010

\section{Keywords:}

A. Dispersion

A. Drying

C. Shrinkage

E. Fiber reinforcement

Internal curing

\begin{abstract}
A B S T R A C T
The objective of this study was to determine how the early-age shrinkage behavior of cementitious materials is affected by the addition of saturated cellulose fibers under sealed and unsealed conditions. The sealed condition simulates autogenous shrinkage exclusively while the unsealed condition introduces drying shrinkage, as well. Although the primary focus was to determine whether saturated cellulose fibers are suitable to mitigate autogenous shrinkage as an internal curing agent, evaluating their effect under drying conditions provided further insight into the overall shrinkage behavior of the composite material. At additions of $1 \%$ by mass of cement, the cellulose fibers were found to show significant drying shrinkage cracking control while providing some internal curing. In addition, early-age shrinkage test results were supplemented with a quantitative measure of fiber dispersion based on comparing theoretical and experimental values of the fiber volume fraction in hardened cementitious samples. Results indicated that improved dispersion leads to improved properties.
\end{abstract}

(c) 2010 Elsevier Ltd. All rights reserved.

\section{Introduction}

In recent years there has been an increase in the application of high-performance concrete (HPC) in the infrastructure due to its superior properties, such as high strength and low permeability, which are achieved through its characteristically dense microstructure. However, self-desiccation leading to autogenous shrinkage has been recognized to be a major shortcoming of HPC [1]. Autogenous shrinkage is bulk shrinkage that is due exclusively to hydration without any influence from the surrounding environment.

Autogenous shrinkage is problematic because it occurs at very early ages, before the concrete has had a chance to develop sufficient structural strength. Unable to resist the tensile stresses, the material will experience internal cracking, increased permeability and become susceptible to water penetration and chemical attack. This inevitably leads to major durability issues. In response, various mitigation strategies have been explored, one of which is internal curing. Internal curing provides additional curing water through the inclusion of water-saturated porous materials, referred to as internal curing agents (IC agents), in the hydrating cement paste. This strategy is effective because the distance that the additional curing water must travel through the dense microstructure to replenish the emptying pores is dramatically reduced

* Corresponding author. Address: 2145 Sheridan Road, Suite A130, Evanston, IL 60208, USA. Tel.: +1 8474917161.

E-mail address: s-kawashima@northwestern.edu (S. Kawashima). compared to traditional external curing methods. Voids that are otherwise difficult to reach are made accessible by well-dispersed IC agents and self-desiccation is averted. Saturated lightweight aggregates (LWA) and superabsorbent polymers (SAP) have been demonstrated to be effective IC agents [2-5].

While it is widely understood that adding cellulose fibers to cement-based systems helps control drying shrinkage cracking and plastic shrinkage [6-9] literature on their effect on autogenous shrinkage is sparse [10]. However, since cellulose fibers can be dispersed in hydrating cement paste and have the capacity to absorb and release water, they may be considered as candidates for internal curing. The obvious advantage of cellulose fibers over LWA and SAP is that they have the additional potential to provide crack resistance. The effect would be similar to a shrinkage reducing admixture (SRA) and PVA fiber blended concrete, as studied by Passuello et al. [11], where the SRA reduces drying shrinkage while the PVA fibers increases crack opening resistance. The focus of this study was to determine the effect of saturated cellulose fiber additions on the early-age shrinkage behavior of cementitious materials, specifically autogenous shrinkage and drying shrinkage cracking.

In addition, a quantitative measure of fiber dispersion was performed and correlated with the shrinkage results. In both applications of internal curing and fiber reinforcement, it is important to achieve good dispersion in the material - a fiber must be at the site of crack initiation and an IC agent must be in the vicinity of hydrating paste. Otherwise the benefits of these inclusions are not fully taken advantage of and any under-reinforced area or fiber clumping will act as a flaw in the material. Past work has shown direct 
correlations between fiber dispersion and performance of fiberreinforced materials [12,13].

\section{Materials and methods}

\subsection{Materials}

The cellulose fibers used in this study are $100 \%$ virgin specialty cellulose fibers with an alkaline resistant coating. They have an average length of $2.1 \mathrm{~mm}$, an average diameter of $16 \mu \mathrm{m}$, and a density of $1.1 \mathrm{~g} / \mathrm{cc}$. Their average tensile strength and elastic modulus are $750 \mathrm{MPa}$ and $8500 \mathrm{MPa}$, respectively. The cellulose fibers are hollow, which allow them to absorb and retain water. Their absorption was determined using a modified water retention value (WRV) test [14] based on the Technical Association of the Pulp and Paper Industry (TAPPI) Useful Method (UM) 256. For cellulose fibers saturated in water for $24 \mathrm{~h}$, the absorption was found to be 1.2 (mass of water absorbed/mass of dry fiber).

Type I ordinary portland cement and tap water were used in all mixes. River sand with the particle size distribution presented in Table 1 was used in mortar and ASTM \#57 limestone [15] was used in concrete. The oven-dry specific gravities of the sand and limestone are 2.66 and 2.65, respectively. A polycarboxylate-based high-range water-reducer (HRWR) was used at various dosages to improve workability.

All paste mixes had a water-to-cement $(\mathrm{w} / \mathrm{c})$ ratio of 0.28 and $1 \%$ addition of superplasticizer by mass of cement. Mortar mixes with a w/c ratio of 0.28 and 0.5 were tested, both of which had a cement-to-sand ratio of $1: 2$. $1 \%$ addition of superplasticizer by mass of cement was used in the $\mathrm{w} / \mathrm{c}=0.28 \mathrm{mix}$ while no superplasticizer was required in the $\mathrm{w} / \mathrm{c}=0.5 \mathrm{mix}$. The composition of the concrete is given in Table 2 , which was specified by the manufacturer of the cellulose fibers as a $30 \mathrm{MPa}$ commercial concrete mix. Although for paste and mortar the dosage of superplasticizer was kept constant between the plain and fiber-reinforced mixes, for concrete it was adjusted to maintain a slump within the range of $10-15 \mathrm{~cm}: 0.34 \%$ by mass of cement for the plain and $1 \%$ for the fiber-reinforced. The slump test was performed in accordance to standard ASTM C143-10 [16]. Cellulose fibers were added by percent mass of cement, measured by dry mass. (Note: In all mixes, the amount of internal curing water introduced by the cellulose fibers was not included in the initial $\mathrm{w} / \mathrm{c}$ ratio. And the cellulose fibers were saturated in water for $24 \mathrm{~h}$ prior to mixing.)

During manufacturing, the cellulose fibers are pressed and rolled out into sheets then cut up into $5 \mathrm{~mm} \times 5 \mathrm{~mm}$ squares. Although this allows for easy handling, they require substantial energy to break up and disperse. For all mixes with a fiber addition, the fibers were premixed with water for 10-20 min in a planetary mixer (small or medium, depending on the size of the batch) to facilitate dispersion. Aside from this preliminary step, the mixing procedures for the mixes with and without a fiber addition were the same and are presented in Table 3. Sufficient periods of mixing were performed to further facilitate fiber dispersion. Paste was

Table 1

Particle size distribution of silica sand.

\begin{tabular}{lc}
\hline Sieve size $(\mathrm{mm})$ & \% passing \\
\hline 9.5 & 100.00 \\
4.75 & 99.31 \\
2.36 & 89.13 \\
1.18 & 74.82 \\
0.6 & 56.70 \\
0.3 & 21.03 \\
0.15 & 2.59 \\
0.075 & 0.59 \\
\hline
\end{tabular}

Table 2

Composition of $30 \mathrm{MPa}$ commercial concrete mix.

\begin{tabular}{ll}
\hline Constituents & Mass per unit volume of concrete $\left(\mathrm{kg} / \mathrm{m}^{3}\right)$ \\
\hline Cement & 333 \\
\#57 limestone & 1092 \\
Sand & 738 \\
Water & 148 \\
Slump & $10-15 \mathrm{~cm}$ \\
\hline
\end{tabular}

Table 3

Mixing procedures.

\begin{tabular}{ll}
\hline Mix & Mixing procedure \\
\hline Paste & (1) Add superplasticizer (and water), mix for 2 min at high speed \\
& (2) Add cement, mix for 1 min at low speed, scrape bowl, mix for \\
& 4 min at high speed \\
Mortar & (1) Add superplasticizer (and water), mix for 2 min at high speed \\
& (2) Add sand, mix for 2 min at low speed, scrape bowl, mix for \\
& 4 min at high speed \\
& (3) Add cement, mix for 1 min at low speed, scrape bowl, mix for \\
3 min at high speed \\
(1) Add superplasticizer (and water), mix for 3 min \\
(2) Add coarse aggregate, mix for 3 min, scrape, mix for 5 min \\
(3) Add sand, mix for 3 min, scrape, mix for 5 min \\
(4) Add cement, mix for 2 min, scrape, mix for 4 min
\end{tabular}

mixed in a small planetary mixer while mortar was mixed in a medium or large planetary mixer, depending on the size of the batch. Concrete was mixed in a large rotary mixer.

\subsection{Amount of saturated cellulose fibers necessary for internal curing}

A relationship was developed by Bentz et al. [17] to calculate how much LWA is needed to supply enough water for internal curing for a given mix. This equation was adopted here for cellulose fibers:

$M_{C F}=\frac{C_{f} \times C S \times \alpha_{\max }}{S \times \phi_{C F}}$

where $M_{C F}$ is the mass of dry cellulose fibers needed for a unit volume of concrete $\left(\mathrm{kg} / \mathrm{m}^{3}\right), C_{f}$ is the cement factor (content) $(\mathrm{kg} /$ $\mathrm{m}^{3}$ ), CS is the chemical shrinkage of cement ( $\mathrm{g}$ of water/g of cement), $\alpha_{\max }$ is the maximum expected degree of hydration of cement, $S$ is the degree of saturation of fiber ( 0 to 1 ), and $\phi_{C F}$ is the absorption of the cellulose fibers ( $\mathrm{kg}$ water $/ \mathrm{kg}$ dry cellulose fibers). With spatial considerations, it is assumed that a mix with a w/c of 0.42 or higher will be able to achieve complete hydration under sealed conditions. Similarly, a mix with a w/c of 0.36 or higher will be able to achieve complete hydration under saturated conditions. Therefore $\alpha_{\max }$ is estimated to be (w/c)/0.36 for $w /$ $\mathrm{c}<0.36$ and $\alpha_{\max }=1$ for $0.36 \leqslant \mathrm{w} / \mathrm{c} \leqslant 0.42$. As determined by the WRV test, $\phi_{C F}$ was 1.2 . CS was taken to be $0.07 \mathrm{~g}$ water $/ \mathrm{g}$ cement because only type I portland cement was used in this study. $S$ was assumed to be 1 because the cellulose fibers were pre-soaked in water for $24 \mathrm{~h}$ prior to mixing, as they were in the WRV test. Lastly, it was assumed that all of the water absorbed by the cellulose fibers was completely desorbed and accessible to the paste.

Fig. 1 shows the relationship between the amount of cellulose fibers necessary as a percentage by mass of cement and w/c ratio. This Fig. is based on plots by Bentz et al. [17] and Jensen and Hansen [18] of water demand of cement versus w/c ratio. Each curve represents a different view for the amount of internal curing water necessary. Both increase linearly up to $\mathrm{w} / \mathrm{c}=0.36$ : higher $\mathrm{w} / \mathrm{c}$ ratios are correlated with higher degrees of hydration and increased chemical shrinkage. However after $\mathrm{w} / \mathrm{c}=0.36$ the two curves di- 


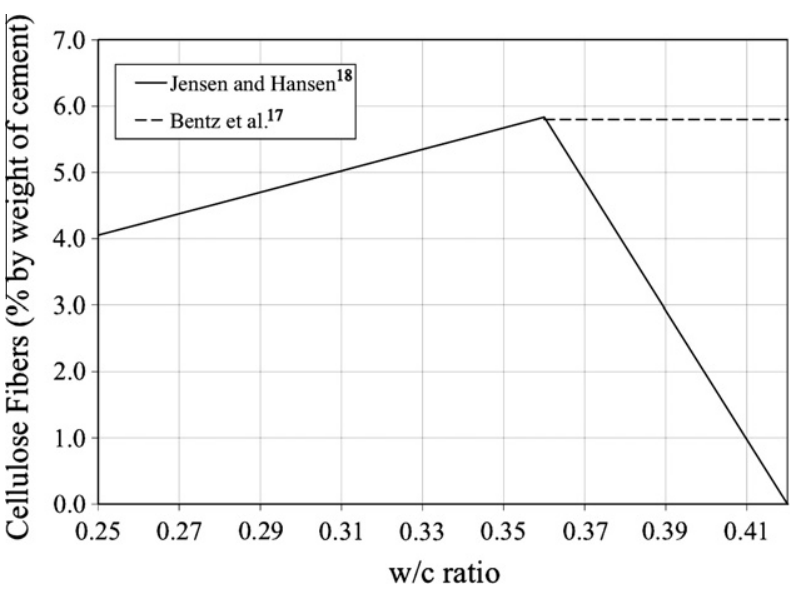

Fig. 1. Theoretical amount of cellulose fibers necessary for internal curing (\% by mass of cement) for a cementitious material with a given $\mathrm{w} / \mathrm{c}$ ratio.

Table 4

Results of slump test on mortar (1\% addition of superplasticizer by mass of cement).

\begin{tabular}{llll}
\hline Mix & Fiber addition & Slump $(\mathrm{cm})$ & Slump flow $(\mathrm{cm})$ \\
\hline Mortar $(\mathrm{w} / \mathrm{c}=0.28)$ & 0 & 23.5 & 39.0 \\
& 1 & 3.5 & - \\
Mortar $(\mathrm{w} / \mathrm{c}=0.5)$ & 0 & - & 68.0 \\
& 1 & 23.5 & 42.0 \\
\hline
\end{tabular}

verge. Bentz et al. [17] proposed that it is necessary to completely compensate for chemical shrinkage in order to keep the pores in the paste saturated. As a result, since $\alpha_{\max }=1$ for $0.36 \leqslant \mathrm{w} /$ $c \leqslant 0.42$, the amount of internal curing water (or cellulose fibers) necessary is the same for mixes with $\mathrm{w} / \mathrm{c}$ ratios within this range. On the other hand, Jensen and Hansen [18] proposed that only enough water to complete cement hydration is required, i.e. enough to yield a w/c ratio of 0.42 (where full hydration is achieved under sealed conditions), so the curve decreases linearly to zero.

Upon looking at Fig. 1 it is apparent that high amounts of fibers are necessary, with fiber additions in the range of $4-5.8 \%$ by mass of cement for $\mathrm{w} / \mathrm{c}$ ratios $0.25-0.36$. This is due to the relatively low water absorption of the cellulose fibers. For the application of internal curing, cellulose fibers introduce a challenge that LWA do not. LWA are typically introduced to the mix by normal aggregate replacement, therefore the change in workability of the mix, if any, is negligible. (For instance, concrete mixes with up to $30 \%$ replacement of normal aggregate by volume with LWA can achieve a slump within $20 \mathrm{~mm}$ of the control mix at the same superplasticizer dosage [19].). On the other hand, cellulose fibers are introduced by addition and have a negative effect on workability.

To illustrate how the addition of cellulose fibers affects workability, a slump test was performed on the mortar mixes (see Section 2.1) based on standards ASTM C143-10 [16] and ASTM C161109b [20] to measure slump and slump flow (horizontal spread), respectively. The results are presented in Table 4. It is apparent that the addition of fibers had a stiffening effect - the $1 \%$ fiber addition decreased fluidity in both $\mathrm{w} / \mathrm{c}$ ratio 0.28 and 0.5 mixes. It was due to this effect that the fiber dosage did not exceed $2 \%$ in the samples tested in this study.

\subsection{Dispersion}

As aforementioned, the $5 \mathrm{~mm} \times 5 \mathrm{~mm}$ pads that the cellulose fibers came in were foreseen to be difficult to break up and disperse. Indeed, visible clumps were present in the cross-sections of some samples, where "clumps" are defined as pads of fibers that were not successfully broken up during the mixing process. Although in previous studies by Rapoport et al. [9] and Akkaya [21] statistical point processes were used to measure dispersion, where each fiber was treated as a point, due to the nature of these clumps comparing volume fractions was believed to be sufficient for the purposes of this study.

Fiber clumping will leave fewer fibers to be singly dispersed throughout the rest of the material, i.e. paste, mortar, or concrete. Therefore, in a material with poor dispersion the volume fraction of fibers in the regions between clumps will be less than the global volume fraction of fibers of the material. This is illustrated in Fig. 2, where $V$ is a representative volumetric element (RVE) of the material, $d V$ is a volume of the RVE without fiber clumping, and $V_{f}$ and $d V_{f}$ are their fiber volume fractions, respectively. If the fibers are poorly dispersed, i.e. fiber clumps are present, then $d V_{f}<V_{f}$. And as the number of clumps in the material approaches zero, $d V_{f}$ should approach $V_{f}$. In this way, comparing $d V_{f}$ against $V_{f}$ can provide a quantitative measure of fiber dispersion. It will be discussed here that $d V_{f}$ may be obtained experimentally through image analysis (IA) and $V_{f}$ may be calculated theoretically.

In order to perform an image analysis, it was first necessary to see the fibers. A method similar to the one developed by Rapoport [22] was used. Dispersion samples were approximately $51 \mathrm{~mm} \times$ $51 \mathrm{~mm}$ in cross-section and $10 \mathrm{~mm}$ in thickness, cut with a band saw from hardened paste, mortar, and concrete with a $1 \%$ addition

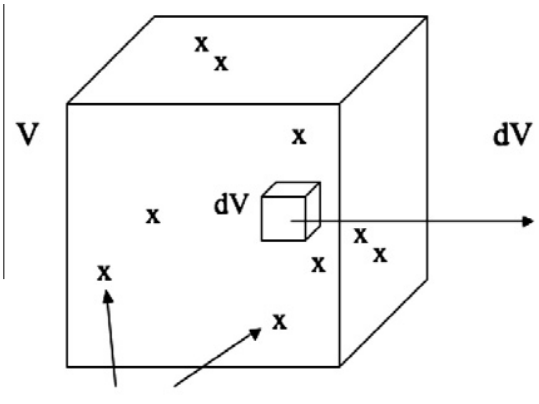

Fiber clumps

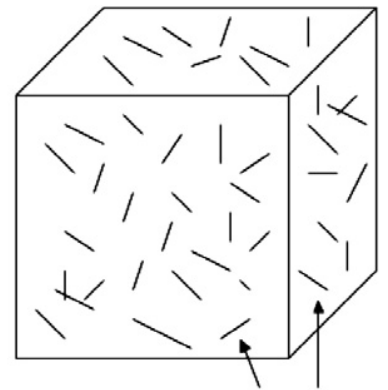

Single fibers

Comparing volume fraction of fibers: 


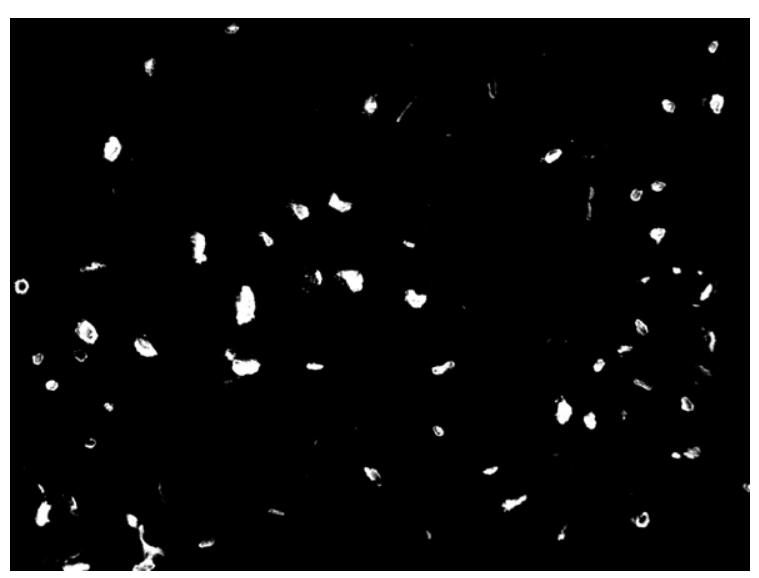

Fig. 3. Binary image of a w/c $=0.28$ paste sample with an area fraction, $A_{f}=0.022$. The image captures an area of $1.8 \mathrm{~mm} \times 1.8 \mathrm{~mm}$.

of cellulose fibers by mass of cement. In obtaining $d V$ it was necessary to avoid any fiber clumps so it was ensured that the cross-sections of the dispersion samples were free of visible clumping. The surface of each dispersion sample was polished successively with 180-grit and 240-grit paper. Once polished, they were treated with a fluorescent brightening agent, which adhered to the cellulose fibers (and not the cement matrix) and allowed them to fluoresce under ultraviolet (UV) light. A layer of the solution was applied to the surface of the polished dispersion samples with a syringe and left for 3-5 min. The samples were then rinsed with water and scrubbed thoroughly dry to ensure no residue of the solution remained. The treated samples were then viewed under a fluorescence microscope at the lowest magnification, where the cellulose fibers fluoresced and were easy to spot against the dark cement matrix. Images were acquired using microscope image processing software - each image captured a $1.8 \mathrm{~mm} \times 1.8 \mathrm{~mm}$ area of the sample (see Fig. 3 ). The images were then converted into binary images from which the area fraction of fibers was calculated using an algorithm.

For a stationary set there is a stereometric relationship [23]:

$V_{f}(x)=A_{f}(x)$

where $V_{V}$ is volume fraction and $A_{A}$ is surface fraction. Using this relationship, the area fraction of fibers obtained from the image analysis was taken to be the volume fraction of fibers. Approximately 100 images were taken over the cross-section of each dispersion sample. The area fraction was calculated for each image and the final volume fraction, $d V_{f}$, was the average. For paste and mortar samples, images were taken over the entire region while for concrete, images were taken around the coarse aggregates due to their comparatively large size. Although the dispersion samples were prepared so that they were free of visible clumping, as aforementioned, any clumps that were overlooked were easily avoided during imaging - clumps would completely overwhelm the area captured in the image $(5 \mathrm{~mm} \times 5 \mathrm{~mm}$ versus $1.8 \mathrm{~mm} \times 1.8 \mathrm{~mm})$, as shown in Fig. 4.

The theoretical fiber volume fraction was calculated based on the original mix composition and the densities of the constituents, i.e. cement, water, sand, and fibers. For concrete, since images were taken around the coarse aggregates the theoretical value was calculated based on the mortar phase of the mix composition. It was important to take into consideration the absorption of the cellulose fibers. As the fibers absorb water, they experience swelling. Because the fibers exist in varying absorptive states in each sample (i.e. fully saturated, partially saturated, or dry) and the degree of swelling was unknown, a lower and upper bound approach was

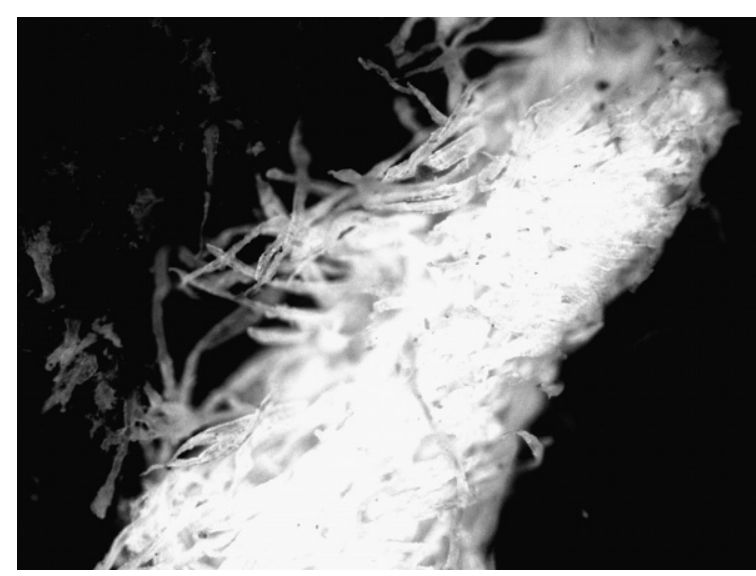

Fig. 4. Binary image of a $w / c=0.28$ paste sample with a fiber clump. The image captures an area of $1.8 \mathrm{~mm} \times 1.8 \mathrm{~mm}$.

employed. The fiber volume fractions for the two extreme states (dry and fully saturated) were calculated. The average of these two values was the final theoretical value. In addition to fiber absorption, chemical shrinkage was also taken into account.

\subsection{Autogenous deformation}

Autogenous deformation was measured using the corrugated tube protocol based on the work of Jensen and Hansen [24] and Sant et al. [25], the setup for which is shown in Fig. 5. It has since become a standard, ASTM C1698-09 [26]. The length change of corrugated polyethylene tubes, approximately $400 \mathrm{~mm}$ in length and $30 \mathrm{~mm}$ in diameter, cast with fresh paste/mortar and sealed with two end caps were monitored. Because the tubes are corrugated, they are much stiffer in the radial direction than they are in the longitudinal direction, thus effectively translating volumetric deformation of the paste/mortar to linear deformation. Paste and mortar with and without a saturated cellulose fiber addition were compared. All mixes had a w/c ratio of 0.28 and a $1 \%$ addition of superplasticizer by mass of cement. The autogenous deformation of samples with cellulose fiber additions of $0 \%, 1 \%$, and $2 \%$ by mass of cement were measured for paste and $0 \%$ and $1 \%$ for mortar. The deformations of two samples were monitored for each mix. Fresh paste/mortar was consolidated by running a vibrating rod along the length of the tube. Each test was run for 7 days in a condition controlled chamber set at $23^{\circ} \mathrm{C}$ and readings were continuously recorded every $15 \mathrm{~min}$ for the entire duration of the test.

All of the readings were zeroed to time of final set because prior to final set (1) the paste is still plastic so what is being measure is essentially chemical shrinkage and (2) the deformation of the polyethylene corrugated tube dominates. The time of final set for each

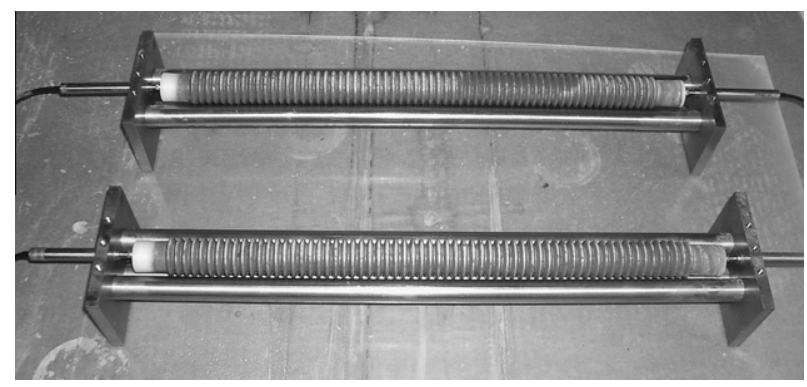

Fig. 5. Setup for the corrugated tube protocol to measure linear autogenous deformation. 
mix was determined by the Vicat Needle Test (a penetration test) in accordance with standard ASTM C191-08 [27].

\subsection{Restrained drying shrinkage}

The restrained ring test based on the standard ASTM C1581-04 [28] was used to monitor drying shrinkage and drying shrinkage cracking behavior, the setup of which is shown in Fig. 6. The steel ring acts as a passive restraint to the surrounding mortar/concrete. As the mortar/concrete undergoes shrinkage, it contracts and applies a uniform stress onto the steel. The strain development within the steel ring may be converted to the circumferential tensile stress within the mortar/concrete using the following equation [29]:

$\sigma=-\varepsilon_{S T} \times E_{\text {Steel }} \times \frac{R_{I C}^{2}-R_{I S}^{2}\left(R_{O C}^{2}+R_{I C}^{2}\right)}{2 R_{I C}^{2}\left(R_{O C}^{2}-R_{I C}^{2}\right)}$

where $R_{I C}=$ inner diameter of concrete ring, $R_{O C}=$ outer diameter of concrete ring, $R_{I S}=$ inner diameter of steel ring, $\varepsilon_{S T}=$ strain in steel ring, and $E_{\text {Steel }}=$ modulus of elasticity of steel. The dimensions of the rings used in this study are given in Fig. 6. In addition to stress development, the age of initial cracking, number of cracks, and crack width development in each ring were also monitored.

Mortar (w/c ratios of 0.28 and 0.5 ) and concrete with and without a $1 \%$ cellulose fiber addition were compared. Fresh mortar/concrete was cast in three layers, each layer consolidated either by manual rodding or a vibrating rod depending on the stiffness of the mix. The rings were then completely sealed and cured at $23^{\circ} \mathrm{C}$ for $24 \mathrm{~h}$ before the forms were removed. Immediately after demolding, a layer of silicone caulking was applied to the top surface while the circumferential surface remained exposed to allow for drying. Two rings were cast for each mix. Each test was run for approximately 30 days in a condition controlled chamber set at $23^{\circ} \mathrm{C}$ and $50 \%$ relative humidity $(\mathrm{RH})$ and readings were continuously recorded every $30 \mathrm{~min}$. After initial cracking occurred,

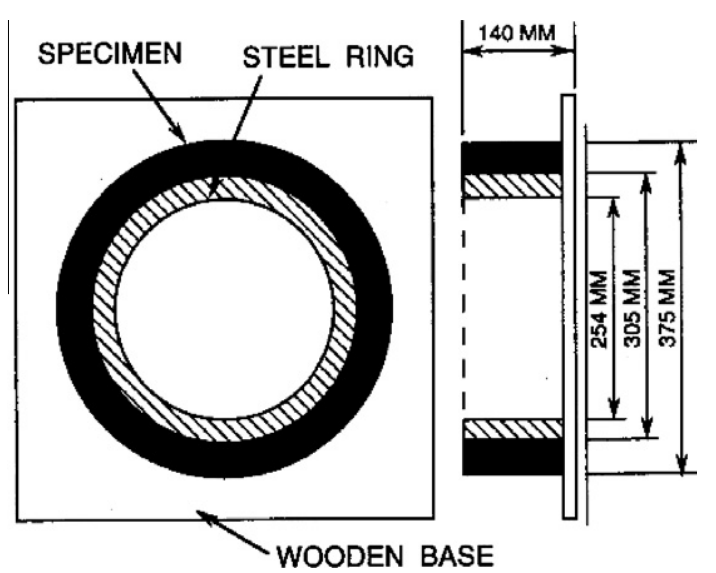

Fig. 6. Setup for the restrained ring test, including ring dimensions [6]. cracks were labeled and measured daily using a microscope with a precision of $2.5 \mu \mathrm{m}$.

\subsection{Unrestrained drying shrinkage}

The free shrinkage prism test according to standard ASTM C157-04 [30] was used to measure the unrestrained drying shrinkage of mortar and concrete specimens. The test monitors the length change of $10.2 \mathrm{~cm} \times 10.2 \mathrm{~cm} \times 30.5 \mathrm{~cm}$ prisms over time and provides a measure of drying shrinkage as linear strain. Immediately after mixing, the mortar/concrete was cast in prism molds in three layers and consolidated either by manually rodding or with a vibrating rod. Each casted mold was then covered with a layer of polyethylene film and allowed to harden. $24 \mathrm{~h}$ after casting the prisms were demolded. Two stubs, which served as the measuring points, were attached to a longitudinal surface $(10.2 \mathrm{~cm} \times 30.5 \mathrm{~cm})$ with epoxy resin and allowed to set for at least $6 \mathrm{~h}$. The gage length was $25.4 \mathrm{~cm}$. The distance between the two stubs was measured daily using a manual strain gage. Three prisms were cast for each mix and monitored. For the duration of the test, the prisms were kept in a condition controlled chamber set at $23^{\circ} \mathrm{C}$ and $50 \% \mathrm{RH}$.

\section{Results and discussion}

\subsection{Dispersion}

For each sample, the fiber volume fraction obtained through image analysis (IA value) was compared against the theoretical value. An IA value less than the theoretical value was an indication of clumping. Table 5 shows the summary of the results. For each material, the IA value fell within the lower and upper bounds, showing the validity of the method. Results indicate that there were varying degrees of clumping in the paste and mortar ( $w /$ $c=0.28$ and 0.5 ) while there was very little to no clumping in the concrete. The results of the dispersion analysis will be referred to throughout the following sections to supplement the results of the shrinkage tests.

\subsection{Autogenous deformation}

The results of the autogenous deformation measurements on the paste and mortar samples are presented in Fig. 7 and Fig. 8, respectively. As expected, the paste experienced greater shrinkage than the mortar due to the absence of sand particles to serve as internal restraints. In both cases it was shown that at high enough additions, the cellulose fibers had a mitigating effect on autogenous shrinkage. For mortar, a $1 \%$ addition of cellulose fibers by mass of cement led to a $13 \%$ reduction in autogenous deformation at 7 days compared to that of the plain. For paste, the curves for the plain and $1 \%$ paste samples were within error, indicating that the $1 \%$ fiber addition did not lead to any measurable reduction in autogenous deformation. On the other hand, the $2 \%$ fiber addition paste showed a reduction of $32 \%$ at 7 days. This may primarily be attributed to the difference in the amount of internal curing water each dosage of cellulose fibers is providing to the hydrating paste.

Table 5

Summary of the dispersion results, comparing theoretical volume fraction of fibers against those obtained through image analysis.

\begin{tabular}{|c|c|c|c|c|c|c|}
\hline \multirow[t]{2}{*}{ Material } & \multicolumn{3}{|l|}{ Theoretical } & \multirow[t]{2}{*}{ Image analysis } & \multirow[t]{2}{*}{ Percent difference } & \multirow[t]{2}{*}{ Clumping (yes/no) } \\
\hline & Lower bound & Upper bound & Average & & & \\
\hline Paste & 0.0164 & 0.0373 & 0.0269 & 0.0214 & -21 & Yes \\
\hline $\operatorname{Mortar}(\mathrm{w} / \mathrm{c}=0.28)$ & 0.0070 & 0.0160 & 0.0115 & 0.0073 & -36 & Yes \\
\hline $\operatorname{Mortar}(\mathrm{w} / \mathrm{c}=0.5)$ & 0.0060 & 0.0139 & 0.0099 & 0.0082 & -17 & Yes \\
\hline Concrete & 0.0057 & 0.0137 & 0.0097 & 0.0098 & 1 & No \\
\hline
\end{tabular}




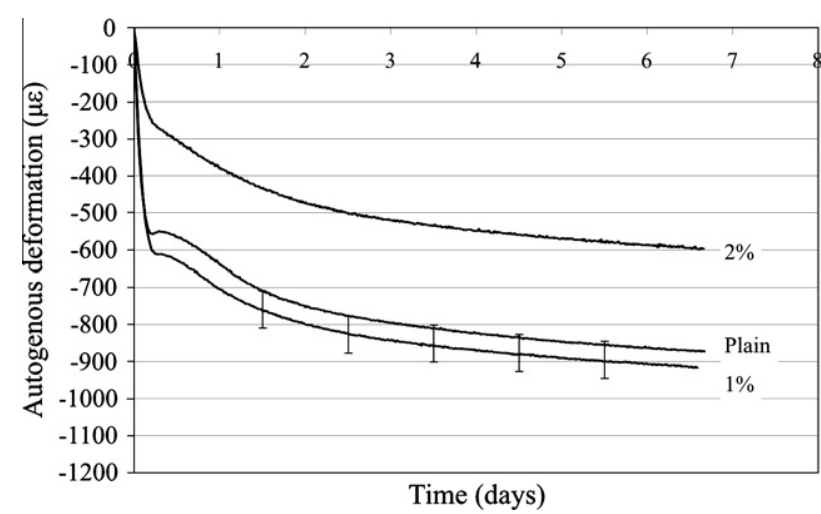

Fig. 7. Linear autogenous deformation of paste samples with and without a cellulose fiber addition. (Zeroed to time of final set.)

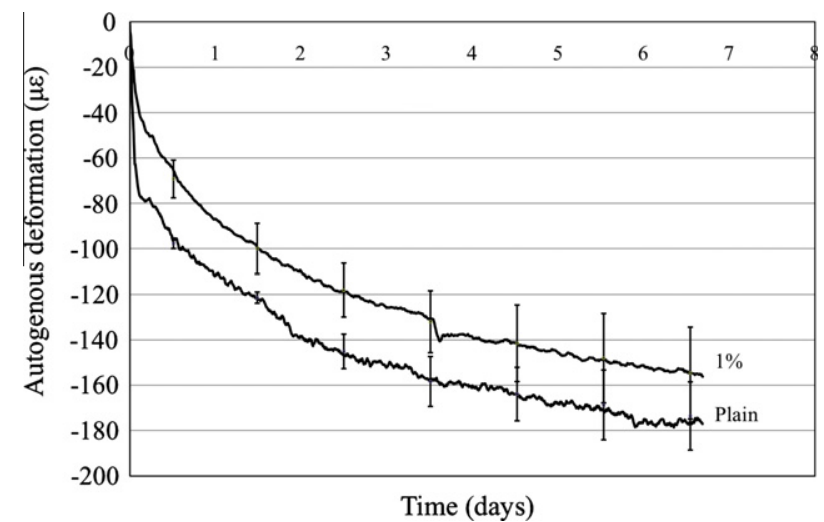

Fig. 8. Linear autogenous deformation of mortar samples with and without a cellulose fiber addition. (Zeroed to time of final set.)

According to Fig. 1 the amount of cellulose fibers necessary to provide enough internal curing water in a paste with a w/c of 0.28 is $4.5 \%$ by mass of cement. This means that a $1 \%$ fiber addition introduces $22 \%$ of the required internal curing water while the $2 \%$ fiber addition introduces twice that, $44 \%$. Still, the $1 \%$ fiber addition should have lead to some mitigation of autogenous shrinkage in the paste samples. The reason for this is unclear but is most likely due to issues of dispersion. Referring back to Table 5, there was fiber clumping present in the $1 \%$ paste. Better fiber dispersion likely would have lead to a measurable effect of internal curing. There may have been fiber clumping in the $2 \%$ paste samples, as well, since similar mixing regimes were followed for both paste mixes. However, the increased amount of internal curing water introduced by the higher fiber addition may have compensated for any issues with dispersion. Dispersion results also indicate that the $w / c=0.28$ mortar had fiber clumping, which suggests that greater mitigation may have been achieved had there been better dispersion.

Although the corrugated tube protocol was performed to evaluate the internal curing effects of the cellulose fibers, their potential reinforcing effect should not be excluded. It is possible that the fibers were controlling autogenous shrinkage-induced internal cracking. If this were the case, the true beneficial internal curing effects of the fibers would have been masked by their reinforcing abilities. Internal cracking would lead to greater deformation as the material slides along the crack surfaces. This can explain why the results did not show reduction in autogenous shrinkage in the $1 \%$ fiber paste samples when compared to the plain samples. The plain samples may have experienced an expansion due to cracking which would underestimate the measured autogenous shrinkage.

\subsection{Restrained and unrestrained drying shrinkage}

The samples are kept sealed for $24 \mathrm{~h}$ before they are exposed to drying conditions. During this time they will experience autogenous shrinkage, particularly the mix with the lower w/c ratio. Therefore it should be noted that autogenous shrinkage will contribute to the measured values in the results presented herein, although it will be referred to as drying shrinkage.

In the $\mathrm{w} / \mathrm{c}=0.5$ mortar (M50), the addition of cellulose fibers did not lead to any reduction in drying shrinkage. Between the plain and fiber-reinforced samples, the strain development under unrestrained conditions and stress development under restrained conditions prior to cracking were similar, shown in Figs. 9 and 10 , respectively. (This was observed in the $\mathrm{w} / \mathrm{c}=0.28$ mortar (M28) and concrete samples, as well.) However, the post-cracking behaviors were markedly different. While in the plain rings the stresses dropped to zero upon initial cracking, the fiber-reinforced rings continued to carry some stress even after the onset of cracking. This indicates that the tensile stress in the mortar was transferred over to the cellulose fibers, which continued to absorb energy. In addition, incrementally decreasing tensile stress in the fiber-reinforced rings signifies multiple cracking, i.e. the cellulose fibers were bridging microcracks to prevent them from widening into macrocracks. This is supported by the $57 \%$ decrease in crack width achieved in the fiber-reinforced rings compared to the plain, as shown in Fig. 11a. Similar behavior was seen in concrete, where the fiber-reinforced rings exhibited a reduced crack of approximately 86\%, shown in Fig. 11c (C1, C2, C3 denote multiple cracks in the ring). This correlates well with the dispersion results, where it was indicated that there was little to no clumping in concrete. In the $\mathrm{w} / \mathrm{c}=0.28$ mortar, however, all of the rings experienced sudden failure, indicated by the stresses dropping past zero. Also, the crack width development in each ring was similar, with initial crack readings at $2 \mathrm{~cm}$ eventually leveling off at $4 \mathrm{~cm}$, Fig. 11b. The exposure to drying in combination with the low $\mathrm{w} / \mathrm{c}$ ratio contributed to this characteristically brittle behavior and the ineffectiveness of the cellulose fiber reinforcement can be traced back to poor dispersion (see Table 5).

The dispersion results indicate that better dispersion was achieved in M50 than in M28. This is in good agreement with the results of the drying restrained shrinkage test, which showed that the fiber addition in M50 lead to significantly more effective crack control. It is suggested that improved fiber dispersion can be achieved in mixes with improved workability. The workability

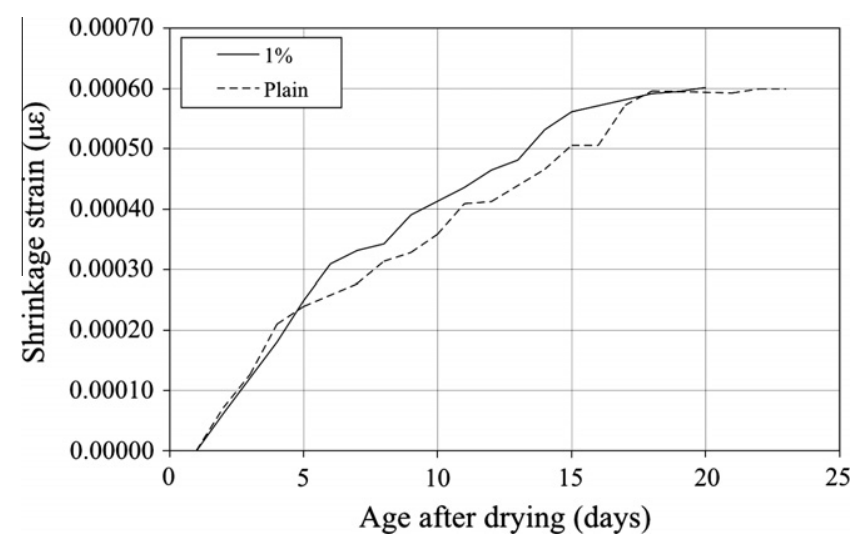

Fig. 9. Unrestrained drying shrinkage of $\operatorname{mortar}(w / c=0.5)$ with and without a cellulose fiber addition. 


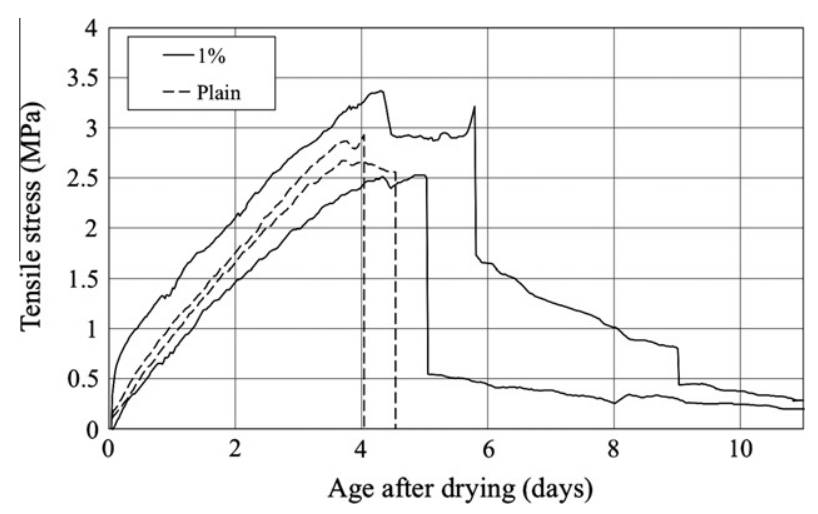

Fig. 10. Restrained drying shrinkage of mortar $(w / c=0.5)$ with and without a cellulose fiber addition.
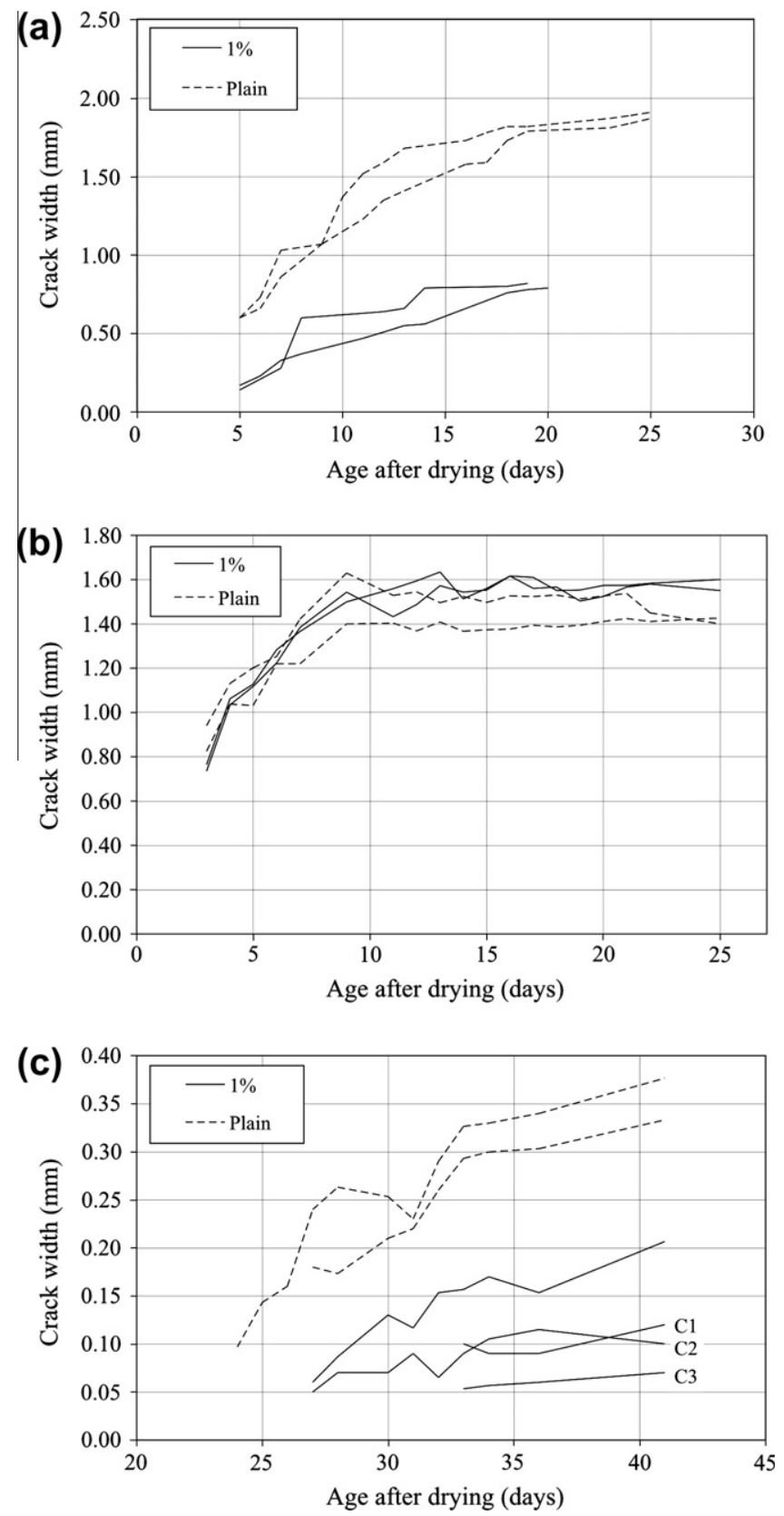

Fig. 11. Crack width development in (a) $\operatorname{mortar}(w / c=0.5)$, (b) $\operatorname{mortar}(w / c=0.28)$, and $c$. concrete rings with and without a cellulose fiber addition. of M50 was significantly better than that of M28 due to the presence of more mixing water (see Table 4), although increasing w/ $\mathrm{b}$ ratio does not necessarily lead to improved dispersion in all cases [31]. Improved dispersion will lead to improved performance of the fibers in both applications of internal curing and reinforcement. Looking back at Fig. 1, according to Jensen and Hansen [18] the amount of fibers required decreases for mixes with a w/ c ratio $0.36-0.42$ and according to Bentz et al. [17] it levels off. In both cases, the implications are good for workability. Furthermore, the best dispersion was achieved in concrete. The large aggregate in the concrete mix most likely helped to break up the fibers more effectively during mixing.

\section{Conclusions}

A quantitative measure of dispersion was performed on hardened paste, mortar, and concrete samples by comparing the theoretical volume fraction of fibers to the volume fraction of fibers obtained through image analysis. There was good correlation between the results of the dispersion analysis and the performance of the samples in the shrinkage tests (i.e. mitigating autogenous shrinkage and drying shrinkage cracking), indicating improved dispersion leads to improved properties.

The results of the autogenous shrinkage tests show that the cellulose fibers have internal curing capabilities. However, due to their relatively low absorption capacity, high amounts of fibers are necessary, to provide enough internal curing water to show a substantial reduction in autogenous shrinkage (for $\mathrm{w} / \mathrm{c}=0.28$, theoretically $4.5 \%$ by mass of cement). This in combination with the adverse effect the cellulose fibers have on workability make them unsuitable for an exclusive application in internal curing.

The cellulose fibers were not found to affect drying shrinkage but they are effective in mitigating drying shrinkage-induced cracking when adequate dispersion is achieved. The fiber-reinforced samples experienced significant reduction in crack width and continued to carry stress after cracking.

The results indicate that improved workability leads to improved fiber dispersion. Furthermore, the best dispersion was achieved in concrete (versus paste or mortar) due to the presence of larger aggregates. This has good implications on the realistic application of the cellulose fibers, since concrete is the most common form of cement-based material in infrastructure.

Possible future work include finding better methods of dispersion for the cellulose fibers used in this study, investigating internal curing using cellulose fibers with higher absorption capacities, and achieving better workability in cellulose fiber-reinforced mixes.

\section{Acknowledgements}

The authors would like to acknowledge the financial support from both the Infrastructure Technology Institute at Northwestern University (Grant DTRT06-G-0051) and Buckeye Technologies, Inc.

\section{References}

[1] Self-desiccation and its importance in concrete technology. In: Proceedings of the 4th international seminar, Gaithersburg, Maryland (USA); 2005.

[2] Bentur A, Igarashi S, Kovler K. Prevention of autogenous shrinkage in highstrength concrete by internal curing using wet lightweight aggregates. Cem Concr Res 2001;31(11):1587-91.

[3] Jensen OM, Hansen PF. Water-entrained cement-based materials: II. Experimental observations. Cem Concr Res 2002;32(6):973-8.

[4] Bentz DP. Internal curing of high-performance blended cement mortars. ACI Mater J 2007;104(4):408-14.

[5] Radlinska A, Rajabipour F, Bucher B, Henkensiefken R, Sant G, Weiss J. Shrinkage mitigation strategies in cementitious systems: a closer look at differences in sealed and unsealed behavior. Transport Res Rec 2008:59-67. 
[6] Sarigaphuti M, Shah SP, Vinson KD. Shrinkage cracking and durability characteristics of cellulose fiber reinforced concrete. ACI Mater J 1993;90(4):309-18.

[7] Soroushian P, Ravanbakhsh S. Control of plastic shrinkage cracking with specialty cellulose fibers. ACI Mater J 1998;95(4):429-35.

[8] Buch N, Rehman OM, Hiller JE. Impact of processed cellulose fibers on portland cement concrete properties. Transport Res Rec 1999;1668:72-80.

[9] Rapoport JR, Shah SP. Cast-in-place cellulose fiber-reinforced cement paste, mortar, and concrete. ACI Mater J 2005;102(5):299-306.

[10] Mohr BJ, Premenko L, Nanko H, Kurtis KE. Examination of wood-derived powders and fibers for internal curing of cement-based materials, Self-desiccation and its importance in concrete technology. In: Proceedings of the 4th international seminar, Gaithersburg, Maryland (USA); 2005. p. 22944.

[11] Passuello A, Moriconi G, Shah SP. Cracking behavior of concrete with shrinkage reducing admixtures and PVA fibers. Cem Concr Compos 2009;31(10):699-704.

[12] Akkaya Y, Shah SP, Ankenman B. Effect of fiber dispersion on multiple cracking of cement composites. J Eng Mech 2001;127(4):311-6.

[13] Ozyurt N, Mason TO, Shah SP. Correlation of fiber dispersion, rheology and mechanical performance of FRCs. Cem Concr Compos 2007;29(2):70-9.

[14] Okubayashi S, Ulrich JG, Bechtold T. A study of moisture sorption and desorption on lyocell fibers. Carbohydr Polym 2004;58(3):293-9.

[15] ASTM C33-07. Standard specification for concrete aggregates. In: ASTM international, West Conshohocken (PA); 2007.

[16] ASTM C143/143M. Slump of hydraulic-cement concrete. In: ASTM international, West Conshohocken (PA); 2010.

[17] Bentz DP, Lura P, Roberts JW. Mixture proportioning for internal curing. Concr Int 2005;27(2):35-40.

[18] Jensen OM, Hansen PF. Water-entrained cement-based materials: I. Principles and theoretical background. Cem Concr Res 2001;31(4):647-54.
[19] Akcay B, Tasdemir MA. Optimisation of using lightweight aggregates in mitigating autogenous deformation of concrete. Constr Build Mater 2009;23(1):353-63.

[20] ASTM C1611/C1611M - 09b. Slump flow of self-consolidating concrete. In: ASTM international, West Conshohocken (PA); 2009.

[21] Akkaya Y. Microstructural characterization of high performance fiber reinforced cement composites. PhD dissertation, Northwestern University Evanston (IL); 2000.

[22] Rapoport, J. Cellulose fiber-reinforced cement paste, mortar, and concrete. PhD dissertation, Northwestern University, Evanston (IL); 2002.

[23] Diggle PJ. Statistical analysis of spatial point patterns. New York: Academic Press Inc.; 1983.

[24] Jensen OM, Hansen PF. Dilatometer for measuring autogenous deformation in hardening portland cement paste. Mater Construct 1995;28(181):406-9.

[25] Sant G, Lura P, Weiss J. Measurement of volume change in cementitious materials at early ages: review of testing protocols and interpretation of results. Transport Res Rec 2006;1979:21-9.

[26] ASTM C1698-09. Standard Test method for autogenous strain of cement paste and mortar. In: ASTM international, West Conshohocken (PA); 2010.

[27] ASTM C191-08. Time of setting for hydraulic cement by vicat needle. In: ASTM International, West Conshohocken (PA); 2008.

[28] ASTM C1581-04. Determining age at cracking and induced tensile stress characteristics of mortar and concrete under restrained shrinkage. In: ASTM international, West Conshohocken (PA); 2004.

[29] Hossain AB, Weiss WJ. Assessing residual stress development and stress relaxation in restrained concrete ring specimens. Cem Concr Compos 2004;26:531-40.

[30] ASTM C157-04. Test method for length change of hardened hydraulic cement mortar and concrete. In: ASTM international, West Conshohocken (PA); 2004

[31] Yang E-H, Sahmaran M, Yang Y, Li VC. Rheological control in production of engineering cementitious composites. ACI Mater J 2009;106(4):357-66. 